\title{
Entre amis : réseaux poétiques dans les ouvrages danois du $17^{\mathrm{e}}$ siècle
}

\section{Joachim Grage}

Traducteur : Pierre-Brice Stahl et Jean-Louis Elloy

\section{(2) OpenEdition Journals}

Édition électronique

URL : https://journals.openedition.org/rbnu/1981

DOl : 10.4000/rbnu. 1981

ISSN : 2679-6104

\section{Éditeur}

Bibliothèque nationale et universitaire de Strasbourg

\section{Édition imprimée}

Date de publication : 1 novembre 2013

Pagination : 44-51

ISBN : 9782859230517

ISSN : 2109-2761

\section{Référence électronique}

Joachim Grage, «Entre amis : réseaux poétiques dans les ouvrages danois du $17^{\mathrm{e}}$ siècle », La Revue de la BNU [En ligne], 8| 2013, mis en ligne le 01 novembre 2013, consulté le 17 mai 2021. URL : http:// journals.openedition.org/rbnu/1981; DOI : https://doi.org/10.4000/rbnu.1981

\section{cc) (†) (2)}

La Revue de la BNU est mise à disposition selon les termes de la Licence Creative Commons Attribution - Pas d'Utilisation Commerciale - Partage dans les Mêmes Conditions 4.0 International. 


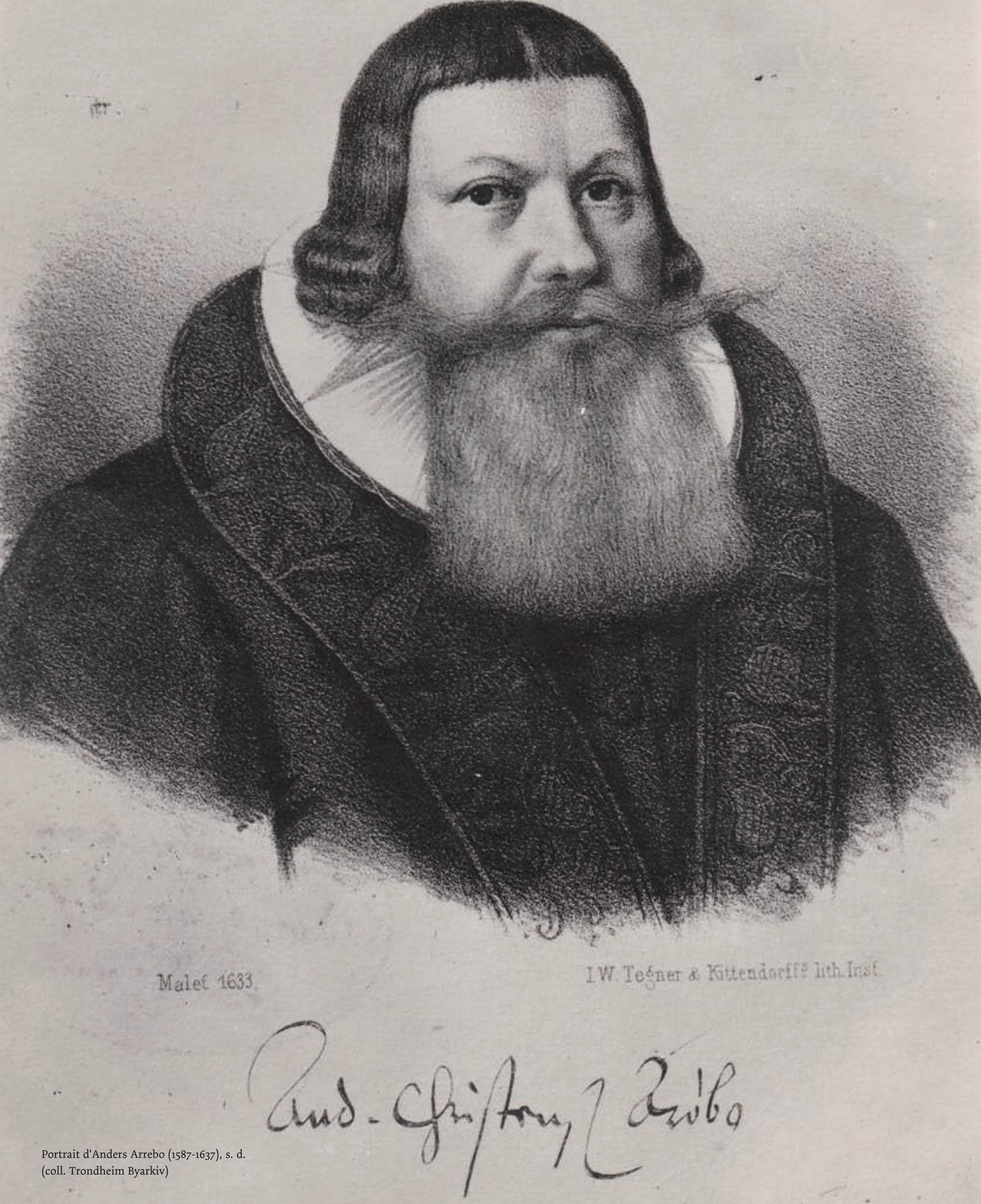




\section{Entre amis : réseaux poétiques dans les ouvrages danois du $I 7^{\mathrm{e}}$ siècle}

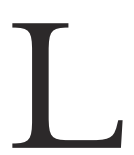

es réseaux sociaux jouent un rôle relationnel déterminant à notre époque. Ils tissent un ensemble de liens d'apparence immatérielle, essentiellement tributaires de l'usage des médias. Sur des plateformes internet comme Facebook ou Twitter, on fait connaissance, on cultive des amitiés. Ces sites s'ajoutent aux autres médias millénaires pour certains - et en complètent l'usage. Les médias de l'écrit restent particulièrement importants dans le développement des relations sociales (de ce point de vue, l'ère d'Internet n'a pas profondément modifié la situation). Il suffit de penser à l'importance que conservent les lettres et les cartes postales dans le maintien des contacts à distance. Et même dans les cas où un échange strictement oral serait possible, on privilégie - hier comme aujourd'hui - les médias de l'écrit dès qu'il s'agit de matérialiser un message, d'en élargir la réception, de le faire partager. La valeur sociale d'une transmission écrite ne réside pas seulement dans son contenu, mais aussi dans l'usage médiatique qu'elle permet.

Tous les spécialistes de littérature ancienne sont constamment amenés à constater que le livre participe d'un réseau social médiatique, et cela bien plus encore autrefois qu'aujourd'hui : actuellement, en offrant un livre, on noue, on entretient ou enrichit des relations avant tout d'ordre personnel. L'ajout d'une dédicace manuscrite donne un caractère d'intime amitié à un produit standardisé. Mais autrefois les vertus sociales du livre s'affichaient plus ouvertement et plus souvent. Jusqu'au $19^{e}$ siècle par exemple, les noms de ceux qui avaient commandé et acheté un livre avant sa publication étaient mentionnés dans l'ouvrage imprimé. Ces listes de souscripteurs sont pour nous riches d'enseignements sur la diffusion des textes concernés et sur le statut social de leurs lecteurs. Quant aux lecteurs contemporains de la publication du livre, ils pouvaient également apprendre quels en étaient les acquéreurs, quelles que fussent leurs motivations : l'intérêt culturel, le souci du prestige social ou un sentiment quelconque d'obligation. Cela faisait partie de leurs habitudes culturelles clairement attestées, comme dans les pays scandinaves jusqu'au début du $19^{e}$ siècle, que de chercher à savoir, à partir des listes de souscripteurs, si le livre se trouvait aussi sur l'étagère de leurs voisins. Cette pratique n'a survécu que dans la "tabula gratulatoria " des mélanges honorifiques (elle a été par ailleurs victime de l'évolution du marché du livre au $19^{\mathrm{e}}$ siècle). Ce répertoire des laudateurs (également souscripteurs) lors des publications réalisées à l'occasion d'un jubilé académique met en évidence le rôle de la mention publique des noms dans l'affermissement des relations sociales.

C'est dans le paratexte que se constitue principalement mais pas seulement - le réseau social du livre. C'est ainsi que Gérard Genette définit les textes périphériques d'un livre, qui permettent précisément au texte principal d'accéder au statut de livre, et qui l'accompagnent dans le volume publié lui-même, comme les titres, les tables des matières, les dédicaces, les avant-propos ou les épigraphes (ce qu'il appelle le péritexte), ou bien les textes publiés autour du livre et dans lesquels l'auteur s'exprime à propos de son œuvre, comme par exemple dans les interviews, les diverses contributions ou les lettres (ce que Genette appelle l'épitexte) ${ }^{1}$.

La fonction sociale du paratexte est évidente dans le cas des dédicaces : elle a pour but de créer ou de renforcer une relation entre l'auteur et le dédicataire. Genette 
fait la différence, à ce propos, entre deux pratiques apparentées, mais distinctes. Il est possible d'une part d'offrir un exemplaire isolé d'un livre à une personne déterminée, à titre de cadeau personnel ou de don honorifique, ce que l'on signale généralement par un insert manuscrit accompagné d'une signature sur la page de garde ou au-dessous du titre intérieur. On peut d'autre part dédier " la réalité idéale de l'œuvre en ellemême $"^{2}$ à autrui, et le faire apparaître dans une dédicace paratextuelle imprimée aux côtés de l'œuvre, dans un texte que tout un chacun pourra lire dans chaque exemplaire du livre. Genette appelle cette deuxième pratique l'acte de "dédier ", afin de la distinguer de l'acte de dédicacer qui ne concerne au sens strict qu'un exemplaire unique. Dédicacer un livre, chacun en principe peut le faire, dans le cadre d'un don la plupart du temps, tandis que le dédier n'est possible que pour l'auteur ou pour ceux qui sont directement concernés par la production du livre - un éditeur ou un traducteur, qui ne peuvent dès lors symboliquement présenter au destinataire que leur propre participation à l'œuvre. Dans les livres des temps modernes, l'acte de dédier est une pratique largement répandue ; généralement il ne se manifeste pas uniquement en une courte phrase ou en un " Pour XY » laconique, mais par des discours développés qui témoignent du degré de gratitude de l'auteur envers son destinataire et qui lui rendent un hommage appuyé. L'acte de dédier une œuvre fait donc apparaître un ordre social hiérarchisé dans lequel les auteurs se placent à un niveau inférieur à celui du dédicataire. Ainsi le fait de dédier un ouvrage établit et concrétise explicitement un rapport d'allégeance, afin de consolider une relation susceptible de garantir de futures faveurs. Le livre devient dès lors l'expression d'un patronage comme il en existe dans la société féodale, et la dédicace a pour fonction d'attester la validité de cet engagement protectoral.

Parallèlement à ce type de dédicaces fondées sur une relation hiérarchique, le livre des premiers temps modernes témoigne également de liens entre personnes d'un même niveau social ou appartenant à une même sphère, sans rapport de dépendance ou d'obligation. Ceuxci s'expriment alors dans des textes d'un auteur différent de celui de l'œuvre publiée, et qui au contraire s'adressent à lui, honorent sa création, et se présentent souvent sous une forme versifiée dans ce que l'on pourrait appeler des hommages poétiques. Si la recherche s'est de façon assez convaincante systématiquement consacrée au paratexte dans les ouvrages des premiers temps modernes, les études littéraires ne se sont guère - voire pas du tout penchées sur la pratique de ces hommages poétiques, sinon, dans le meilleur des cas, de façon indirecte, en évoquant leur lien avec l'édition ou les commentaires des textes de cette époque. Il n'est d'ailleurs pas étonnant qu'ils aient retenu l'attention sur ce dernier point car ils posent en effet un problème éditorial. En atteste par exemple l'édition scientifique des œuvres du plus célèbre poète baroque danois, Thomas Kingo (1634-1703), surtout connu pour ses hymnes religieuses. L'édition Samlede Skrifter (1939-1975) établie par Hans Brix, Paul Diderichsen et F. J. Billeskov Jansen contient à la fois les hommages poétiques écrits par d'autres auteurs pour l'ouvrage de Kingo et ceux de Kingo lui-même, rédigés pour d'autres œuvres et auteurs. Les poèmes en hommage à Kingo se présentent ainsi dans le contexte pour lequel ils ont été conçus, et font apparaitre dans l'édition moderne comme dans l'originale leur nature de paratexte grâce à l'agencement textuel. Ainsi les hommages poétiques pour la deuxième partie de l'Aandelige Siunge-koor (Chant choral sacré, un recueil de chants et de poèmes sacrés publié par Kingo en 1681) se situent à la même place que dans l'édition d'origine, c'està-dire entre l'imprimatur du roi et la table des matières. Sous le titre commun de In Chorum Sacrum Thomce Kingo sont rassemblés deux poèmes en danois et quatre en latin de cinq auteurs danois qui célèbrent Kingo en tant que poète religieux ${ }^{3}$. Par contre, les hommages poétiques de Kingo ont été inclus dans le volume consacré aux pièces diverses. Un tel choix éditorial a également pour but de nous documenter le plus exhaustivement possible sur l'œuvre de Kingo, mais dans une perspective intratextuelle et non plus dans celle du contexte de leur publication originelle : ces hommages poétiques se retrouvent dès lors présentés à la suite dans un ordre chronologique ; si l'on veut savoir à quel ouvrage ils sont destinés, il faut se reporter au commentaire, qui offre par ailleurs l'avantage de nous donner un aperçu plus général sur les pratiques de l'hommage poétique ${ }^{4}$. Et si l'on cherche alors à connaître l'identité de ceux qui ont écrit des hommages poétiques à Kingo et celle de ceux pour qui Kingo luimême a composé de tels poèmes, il ressort que le groupe d'auteurs qui pratiquent entre eux cet échange n'est pas socialement homogène : il s'agit de prêtres, d'employés, de lettrés de toute sorte, dont presqu'aucun n'a un statut social équivalent à celui de Kingo, devenu en 1677 évêque de l'île de Fionie et anobli deux ans plus tard, mais les textes laissent entendre qu'une communication d'égal à égal reste toujours poétiquement possible. Deux des auteurs qui ont composé des hommages poétiques pour la 


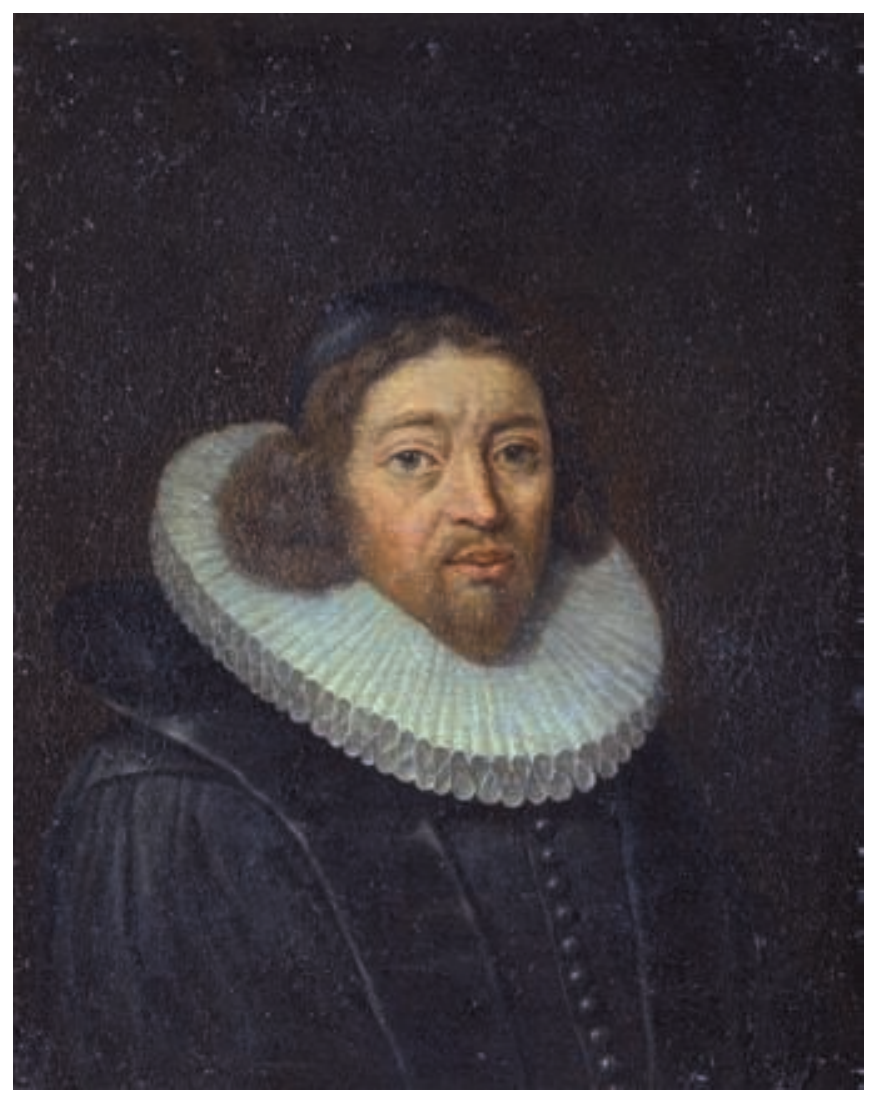

Portrait de Thomas Kingo (1634-1703), peint aux environs de 1670 (coll. Nationalhistoriske Museum, Frederiksborg)

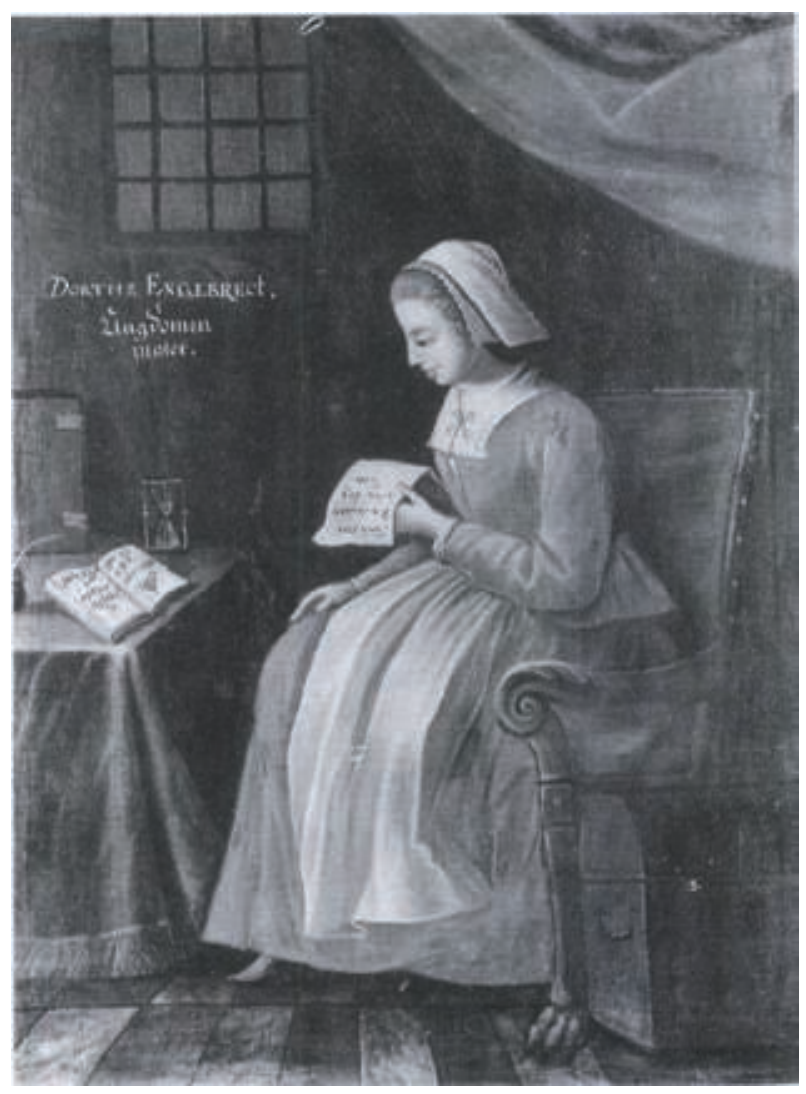

Portrait de Dorothe Engelbretsdatter

(1634-1716 ; coll. Nationalhistoriske Museum, Frederiksborg) 
deuxième partie de l'Aandelige Siunge-koor ont également été gratifiés de tels textes par Kingo. Ce qui cimentait l'union de ces auteurs, c'était une connaissance partagée et approfondie du latin, des études communes à l'université : ils appartenaient tous à la " res publica litteraria ", cet Etat virtuel des intellectuels européens dans lequel ni la catégorie ni l'origine sociales n'avaient d'importance tant que l'on évoluait dans la sphère du savoir ${ }^{5}$. De fait, dans les poèmes eux-mêmes, il n'est jamais question de hiérarchie sociale : seul Jens Pedersson (1639-1704), pasteur de l'église de Dalum sur l'île de Fionie, s'adresse respectueusement à son supérieur dans un poème en utilisant la formule " Wohl-edler Bischof " 6 .

Cet échange entre intellectuels impliquait tout naturellement l'usage de la langue latine. L'humanisme européen l'avait déjà consacrée tant comme langue de communication que comme medium de l'«amicitia», de l'amitié entre égaux. Mais Kingo luimême composa ses hommages poétiques en danois, à une exception près : pour Friedrich Brandt (1632-1691), prévôt de la communauté danoise Nykøbing/Falster qui publia en 1685 une traduction allemande d'hymnes religieuses de Kingo, il écrivit en latin, car bien que le destinataire eût bien évidemment compris le texte, celui-ci serait resté inintelligible pour ses lecteurs allemands.

L'un des hommages poétiques de Kingo mérite d'être signalé pour sa singularité : celui composé en 1685 pour le recueil de poèmes Sicelens Taare-Offer (Les Larmes sacrificielles de l'âme) de la poétesse norvégienne Dorothe Engelbretsdatter (1634-1716). Les échanges poétiques étaient pratiqués entre hommes ; les femmes en étaient exclues, ne serait-ce que parce qu'elles ne jouissaient pas de la formation intellectuelle nécessaire et qu'elles n'avaient pas appris le latin. Mais Kingo loue la poétesse, la gratifie du titre de "Danne-Qvinde » (femme de culture) et lui accorde explicitement une place d'honneur dans la confrérie des poètes (évidemment masculine) ; en lui adressant un poème honorifique, il l'intègre même au prestigieux réseau des intellectuels dont cette pratique constitue précisément le signe de reconnaissance.

De tous les livres de la littérature scandinave, c'est celui intitulé Hexaemeron Rhythmico-Danicum. Det er: Verdens Første Uges Sex Dages proectige og maectige Gierninger (Les Ma- gnifiques et puissants actes des six jours de la première semaine du monde) qui contient l'apparat d'hommages poétiques le plus important. Il s'agit d'une épopée de la Création sur le modèle de celle de Guillaume de Salluste du Bartas (15441590), dont le poème épique encyclopédique La Sepmaine, ou Création du Monde (1578) fut l'un des plus grands succès de l'édition pendant la Renaissance française. Cette œuvre suscita un ensemble d'imitations plus ou moins fidèles, publiées dans de nombreuses langues européennes. Ces épopées de la Création s'appuient toutes sur le récit de la naissance du monde en six jours, qu'elles réécrivent à la lumière des connaissances contemporaines sur la nature et l'être humain. Quand parut l'épopée danoise, son auteur Anders Arrebo (1587-1637) était déjà mort depuis longtemps. Son fils Christen Arrebo fit éditer l'œuvre de son père, dont beaucoup de gens cultivés connaissaient déjà l'existence et que l'on avait déjà lue par extraits, par le biais de citations traduites en vers danois par Hans Mikkelsen Ravn en 1661 et publiées à Copenhague. Cette œuvre répondait à des mobiles politiques définis : le chancelier danois Christen Friis avait demandé à Anders Arrebo, dont la carrière ecclésiastique avait connu de sévères échecs

(il avait été rétrogradé de la dignité d'évêque de Trondheim et relégué comme pasteur dans une paroisse de la province danoise), d'écrire cette épopée afin de magnifier les richesses de la langue danoise et de la hisser au niveau des autres langues littéraires européennes.

Gérard Genette a comparéles paratextes à des seuils qui permettent au lecteur d'entrer dans l'espace intérieur du livre $^{7}$. Dans le prolongement de cette image, on pourrait également comparer les nombreux paratextes de l'Hexaemeron à une enfilade de portails baroques d'apparat; avant que le chant poétique n'entame le récit de la Création, ce ne sont pas moins de 66 pages de paratextes que le lecteur doit parcourir ou feuilleter : la page de titre, l'imprimatur du roi, une dédicace de l'œuvre (" dedicatio ») par l'éditeur Christen Arrebo pour le roi, et entre le préambule de Christen Arrebo adressé aux lecteurs et la Fortale til Skaberen (l'avant-propos adressé au Créateur) d'Anders Arrebo, pas moins de dix-sept éloges et hommages poétiques de quinze auteurs différents, qui occupent à eux seuls 42 pages $^{8}$. Cet enchaînement complexe de paratextes crée un espace polyphonique très riche : nombreux sont les lo- 


\section{HEXAEMERON \\ RHYTHMICO-DANICUM.}

\section{Det ex:}

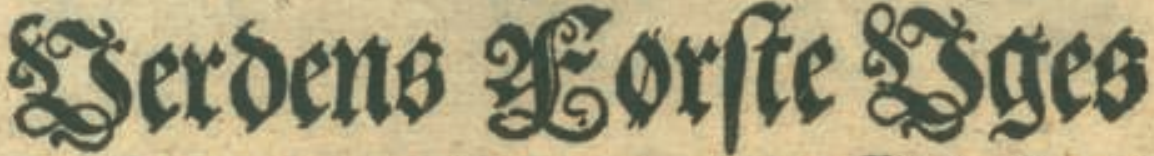

Eer Dages practige or mertige Giterninger/

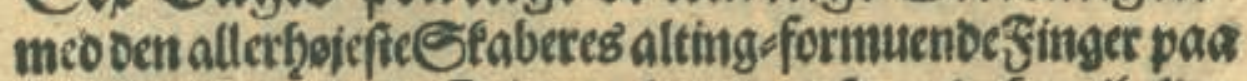
oet allermefierligfie fiabte oc beredoe; 5 ans ubegribclige

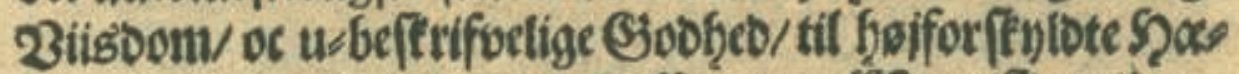
Det oc 2Ere/oc bृans abeligfec Sreature S2Rennifect/til et

ret Surfum corda, Sielsoc 2infitt o oploftelfet.

or Staberens bellige of fertige 2 : fitulfe;

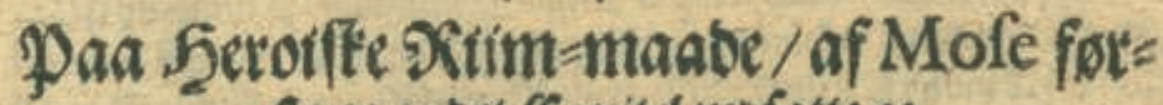
fic of aubet Sapitel/ ub fatte of uboragne

\section{af

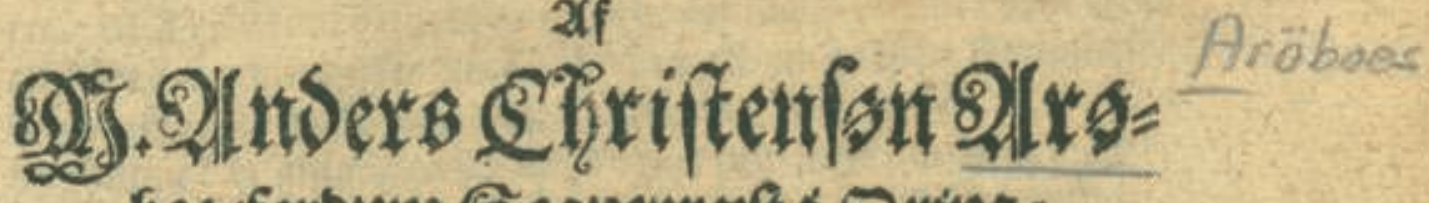 boe/foroum Eognepraft $t$ Dring= borg. \\ Pfalm CXI.}

Store ere Dine Sirerninger ô ŞGrre/ bquo bennem acter/ feer fin Siertens \&ufi ber paa.

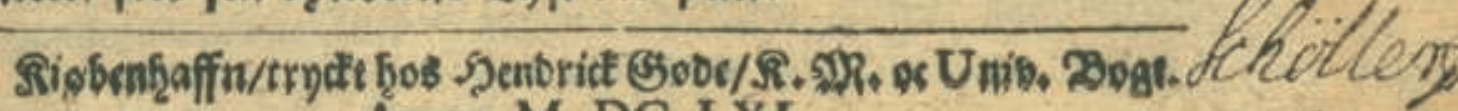
ANNo M DC LXI. 


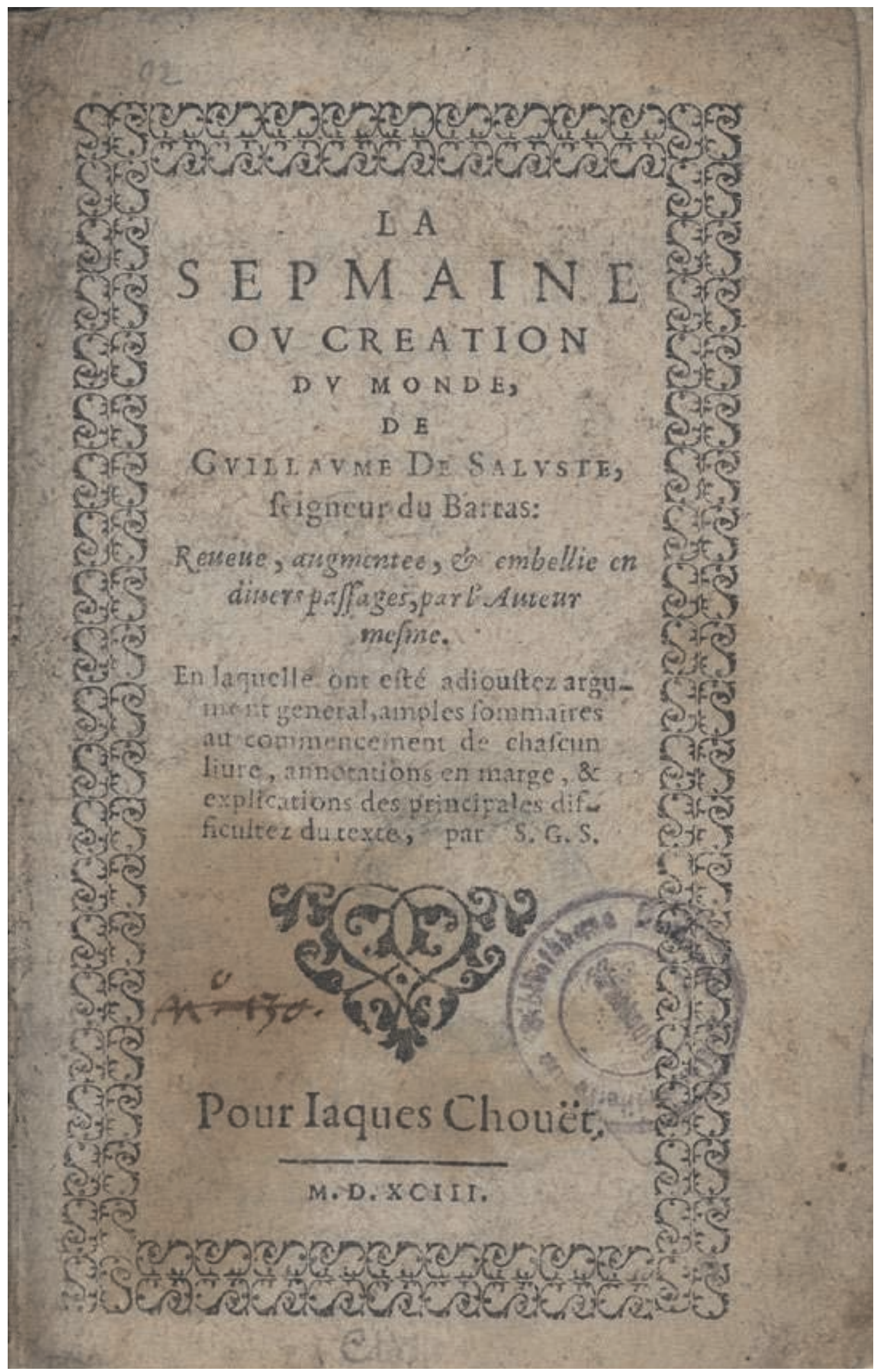

Page de titre de La Sepmaine ou Création du monde de Du Bartas

(édition de 1593 ; coll. BNU) 
cuteurs et destinataires - du roi jusqu'à Dieu, en passant par le poète, l'éditeur et le lecteur, le tout écrit en prose ou en vers à la métrique très variée, en danois ou en latin. Cette richesse paratextuelle fait clairement apparaître l'importance que cette œuvre littéraire d'Anders Arrebo devait revêtir aux yeux de ses contemporains. Dans le même temps, les dédicaces et les hommages poétiques confèrent au livre une valeur mémoriale qui permet à l'auteur d'acquérir une stature historique ; à la différence des hommages poétiques de et pour Thomas Kingo, ces textes ne participent pas à une interaction sociale, mais bien à une entreprise de pérennisation; ils n'ouvrent pas un dialogue mais relèvent d'une culture commémorative dont le but est de hisser Arrebo au rang des grands poètes anciens et de confirmer que l'œuvre du "Arctoi Maronis " (le Virgile nordique) ou du " Danici Nasonis " (l'Ovide danois) ${ }^{9}$ se situe à l'égal des grandes épopées classiques.

Si de nombreux textes sont écrits en latin (dont deux discours en prose qui rendent hommage aux contributions littéraires d'Arrebo), cela tient à ce que les discours savants sur la littérature et ses formes n'avaient pas encore adopté la langue populaire et qu'Arrebo fut l'un des premiers auteurs qui transposa la versification latine en danois : les premières sections de l'Hexaemeron sont composées en hexamètres avec rimes intérieures et finales. Pour un sujet aussi noble que la création du monde, c'était alors le mètre le plus noble que l'on pouvait concevoir. Certains des hommages poétiques danois reprennent ces vers difficiles à adapter dans cette langue (même en renonçant aux rimes) et en optant pour cette forme rendent honneur à Arrebo. En faisant preuve d'un souci formel aussi aigu de la prosodie, en utilisant une telle variété de vers et de strophes dans leurs hommages poétiques, ils font d'Arrebo le père de l'art poétique danois et l'instituent comme fondateur d'une tradition qu'ils s'engagent eux-mêmes à poursuivre. Cela se remarque particulièrement dans les publications de Hans Mikkelsen Ravn (1610-1663), un ami d'Arrebo qui dans les paratextes rend honneur à son œuvre d'une manière aussi bien théorique que pratique. Par deux fois il contribue à cette célébration : en premier lieu dans une section de son poème didactique écrit en latin, Ex rhythmologica Danica (1649), où il détaille tout ce que la littérature danoise doit à Arrebo, et en second lieu dans une suite de cinq poèmes composés en mètres divers, qui reprennent précisément des formes poétiques qu'Arrebo avait utilisées dans son Hexaemeron et ses autres poèmes. Il montre ainsi comment la langue danoise peut être façonnée si l'on suit le chemin pris par cet écrivain.

Mais alors même que les auteurs célèbrent la contribution d'Arrebo à la fondation d'une littérature en langue danoise, liée aux modèles antiques, le média par lequel ils procèdent à cet éloge entre déjà dans sa phase crépusculaire. Avec la fin de la littérature baroque disparaît la pratique de l'échange des hommages poétiques à l'occasion d'une publication éditoriale. C'est uniquement dans un cadre privé que cet usage perdurera, sous la forme de généalogies manuscrites et de livres d'amitié ; les hommages poétiques disparaissent des livres imprimés au cours du $18^{\mathrm{e}}$ siècle. De nombreuses raisons peuvent expliquer ce phénomène : d'une part le déclin de la poésie de circonstance dont le statut de vraie poésie n'est plus reconnu, d'autre part le développement d'une littérature toujours plus consciente d'elle-même, et qui tire de plus en plus sa légitimité de sa propre matière sans avoir besoin d'un paratexte. Mais surtout, la conception de l'amitié connaît alors une véritable transformation sous l'emprise du mode de vie bourgeois qui la cantonne dans une sphère privée et intime. A notre époque toutefois, un nouveau renversement semble se produire, et cela surtout dans les médias où se constituent les réseaux sociaux. Les liens de l'amitié cherchent à nouveau à se déployer dans un espace public et à être plus largement partagés. Les utilisateurs de Facebook communiquent spontanément leurs contacts sociaux et ouvrent à autrui la communication qu'ils ont avec leurs " amis ". Peut-être les conditions sont-elles aujourd'hui réunies pour que l'hommage poétique renaisse sous une nouvelle forme.

\section{Joachim Grage}

(traduction Pierre-Brice Stahl et Jean-Louis Elloy)

\section{Notes}

1 - Cf. Genette, Gérard, Paratexte. Das Buch vom Beiwerk des Buches. Avec une préface de Harald Weinrich. Traduit du français par Dieter Hornig. Frankfurt-a.-M. : Suhrkamp, 2001, p. 9-21

2 - Ibidem, p. 115

3 - Cf. Kingo, Thomas, Samlede Skrifter, vol. 3. Copenhague : Munksgaard, 1939, p. 141-150

4 - Cf. Kingo, Thomas, Samlede Skrifter, vol. 1. Copenhague : Munksgaard, 1945, p. 247-259

5 - Cf. Neumeister, Sebastian et Wiedemann, Conrad (éd.), Res publica litteraria. Die Institutionen der Gelehrsamkeit in der frühen Neuzeit. Wiesbaden : Harrassowitz, 1987 (= Wolfenbütteler Arbeiten zur Barockforschung, 14)

6 - Kingo, Samlede Skrifter, vol. 3, p. 147

7 - Cf. Genette, op. cit., p. 10

8 - Les références de pages correspondent à l'édition d'origine (Kopenhagen : Hendrick Gøde, 1661). Le texte est cité d'après l'édition suivante : Arrebo, Anders, Samlede Skrifter. Ed. Det Danske Sprogog Litteraturselskab. Vol. 1: Hexaëmeron. Leijlighedsdigte. Breve. Ed. Vagn Lundgaard Simonsen. Copenhague : Munksgaard, 1965.

9 - Cf. Thomas Bang (16oo-1661) dans sa laudatio latine. Voir Arrebo, Samlede Skrifter, vol. 1, p. 16 